Sina Varahram

Peyman Jalali

Morteza H. Sadeghi

Saeed Lotfan

https://doi.org/10.21278/TOF.43303

ISSN 1333-1124

eISSN 1849-1391

\title{
EXPERIMENTAL STUDY ON THE EFFECT OF EXCITATION TYPE ON THE OUTPUT-ONLY MODAL ANALYSIS RESULTS
}

\begin{abstract}
Summary
Output-only Modal Analysis (OMA) has found extensive use in the identification of dynamic properties of structures. This study aims to investigate the effect of excitation force on the accuracy of modal parameters. For this purpose, the modal parameters of a simply supported beam are obtained through the Experimental Modal Analysis (EMA) and the OMA method using three different types of artificial and natural excitations, namely a shaker, acoustic waves, and environmental noise. Frequency Domain Decomposition (FDD) technique is used to identify dynamic characteristics. Finally, these results are compared with those obtained by the analytical method and the EMA method. The results demonstrated the following: 1) Acoustic excitation presents the natural frequencies with the smallest errors in comparison with the analytical results. 2) Inaccuracy is observed at certain natural frequencies during the excitation with a shaker with respect to the connecting point between the shaker and the beam. 3) Modal Assurance Criterion (MAC) showed that the mode shapes extracted by the acoustic excitations are more similar to the analytical results.
\end{abstract}

Key words: $\quad$ Vibration, Output-only Modal Analysis, Experimental Modal Analysis, Frequency Domain Decomposition, Singular Values

\section{Introduction}

Dynamic analysis is one of the essential stages in the design, manufacture, and maintenance of structures, but many problems can be faced given the unavailability of analytical solutions to the equations governing the dynamics of complex structures with different boundary conditions and loading procedures. Furthermore, errors such as the use of inappropriate assumptions and theories make the simulation unreliable; therefore, modal analysis methods have been substantially improved to achieve the dynamic characteristics of structures. In addition, results of experimental modal tests can be useful for verifying the precision and accuracy of numerical and theoretical methods [1]. In modal analysis, one can generally use two methods to identify the vibrational properties of a system:

1) Modal analysis which employs the excitation and response measurements [2].

2) Modal analysis which employs only the response measurement [3]. 
In the first method, known as Experimental Modal Analysis (EMA), the system is affected by a specific and controllable excitation. In order to achieve the dynamic characteristics of a system using this method, the measured response and excitation data of the test are processed in the time or the frequency domain based on different methods such as the Least Squares Complex Exponential (LSCE) method [4], the Ibrahim Time Domain method [5, 6], and the Rational Fraction Polynomial (RFP) method [7].

The second method, called Output-only Modal Analysis (OMA), is a relatively new and improved approach which has recently been advanced. In this method, the system can be excited by various types of forces to be analysed, without the necessity to measure theses forces. This advantage of the method makes this method superior to the EMA method. According to the source of excitation, a system can be excited either artificially or naturally. In artificial excitation, the system can be affected by a specific excitation, just like in the EMA method, and modal parameters can then be approximated by the measured responses. However, in natural excitation, the vibration is investigated in the operating condition, such as measuring the vibrations of a turbine in operation or vibrations in the body or other parts of a vehicle moving on a rough road. In this type of excitation, the system can be affected by different unknown sources of excitation, and the approach is therefore called Operational Modal Analysis.

The advantages of operational modal analysis are as follows: 1) Huge structures and constructions, such as bridges and buildings, which cannot easily be excited by artificial and controllable forces, can be excited by forces from natural sources such as wind, acoustic waves, and traffic loads. In mechanical systems such as turbines and vehicles, exciting forces can also be caused by aerodynamic and engine loads, as well as by road and rail traffic loads. 2) The operational modal analysis would achieve modal parameters during work. Even if the system is placed in laboratory conditions, the workplace excitation cannot be exactly simulated. One of the main disadvantages of the OMA method is the existence of high noise which can significantly affect the quality of the results.

Estimation of modal parameters via OMA can be accomplished in the time or the frequency domain just like in EMA; different methods such as Natural Excitation Technique $[8,9]$, Stochastic Sub-space Identification (SSI) [10], and Frequency Domain Decomposition (FDD) $[11,12]$ can be used for this purpose.

The OMA methods in the frequency domain are defined based on the relation between the input and output power spectral densities (PSD) [13]. The peak picking technique is the most efficient method in the frequency domain; in this method, the natural frequencies are obtained directly by acquiring data from the peaks of the PSD diagram [14]. If the system contains separated modes, peak picking is an acceptable method for determining its modal parameters [15]. Brincker et al. offered the FDD method to identify the modes of a system, especially in a noisy condition or modes that are close to each other [11]. In this method, the singular values of PSD at different frequencies are used to determine the modal parameters. The Enhanced Frequency Domain Decomposition (EFDD) has also been defined to approximate the damping ratio $[16,17]$.

In recent years, some researches have been carried out using the OMA method and the FDD technique for extracting modal parameters. Zhang et al. made some changes to the FDD theory and described the method of Frequency Spatial Domain Decomposition (FSDD) [18]. Magalhães et al. compared modal damping ratios which were approximated based on free vibration and ambient tests [19]; the SSI and FDD techniques have been used to identify modal damping ratios in ambient tests. Yan et al. proposed the Power Spectrum Density Transmissibility (PSDT) for other excitations beside the white noise [20]. Araújo et al. implemented Singular Value Decomposition (SVD) on PSDT to reduce extra non-physical 
poles [21]. Yan et al. proposed an enhanced PSDT approach which identified the natural frequencies using the Least Squares Complex Frequency domain (LSCF) and extracted mode shapes using SVD on the PSDT matrix [22]. Kudu et al. compared the natural frequencies and the quality of modal damping ratios obtained by EFDD and SSI, taking into account the measurement duration, the frequency range, and the sampling rate [23]. The change in natural frequencies was shown to be negligible, but the modal damping ratios were changed under different analytical conditions. Wang et al. implemented the FDD and FSDD methods by strain measurements [24]. They adopted SVD, the least square fitting approach, and the power spectrum enhancement technique to acquire modal parameters. Malekjafarian et al. implemented Short Time Frequency Domain Decomposition (STFDD) on the signals that had been measured by accelerometers installed on a vehicle moving across a bridge to extract the mode shapes of the bridge [25].

As discussed above, there are many advanced methods for experimental investigation of vibrational behavior of structures, while the OMA method is preferred when exciting the base structure under a controlled condition and when the recording of the input is not possible. In this respect, the OMA method has been developed but the main question to be dealt with in this method is the effect of unmeasured input excitation type on the performance of the overall method. Accordingly, in this paper, the influence of the type of excitation on the results of the OMA method is investigated from the perspective of the natural or the artificial excitation. Considering the system investigated in this paper, which is a simply supported beam, and the methods used for the above-mentioned purposes, the related theoretical foundations are described in section 2 . In section 3, the laboratory testing conditions are explained, and in section 4, the results of the tests are illustrated, discussed and compared with each other, which is finally followed by the conclusion in section 5 .

\section{Theoretical foundations}

\subsection{Beam theory}

For an Euler-Bernoulli beam with a uniform cross section, which has free vibration, a governing equation of transverse motion can be represented as [26]:

$$
c^{2} \frac{\partial^{4} w}{\partial x^{4}}+\frac{\partial^{2} w}{\partial t^{2}}=0
$$

where $c=\sqrt{\frac{E I}{\rho A}}$.

Considering the boundary conditions of the system investigated in this study (simply supported beam) and solving the free vibration of Eq. (1), the $n$th vibrational natural frequency becomes:

$$
\omega_{n}=(n \pi)^{2}\left(\frac{E I}{\rho A L^{4}}\right)^{1 / 2} \quad n=1,2, \ldots
$$

In addition, the vibrational normal modes are given by:

$$
W_{n}(x)=\sin \frac{n \pi x}{l} \quad n=1,2, \ldots
$$

In practice, only the modes associated with significant mass participation are required to represent and investigate the response of a system in a desired frequency range. Basically, the 
modal participation factor provides a measure of the energy contained in each mode as it represents the amount of system mass participating in that mode and provides an approach to quantify the importance of a vibration mode. Therefore, a mode with a large effective modal mass (which is directly related to the modal participation factor) shows significant contribution of the system's response to that mode [27].

Considering the normalized mode shapes with respect to the mass matrix, the modal participation factor $\Gamma_{n}$ and the effective modal mass $m_{\text {eff }, n}$ of the $n$th mode of the simply supported beam can be defined as:

$$
\begin{aligned}
& \Gamma_{n}=-\sqrt{2 \rho A L}\left[\frac{1}{n \pi}\right][\cos (n \pi)-1] \quad n=1,2,3, \ldots \\
& m_{\text {eff }, n}=\left[\Gamma_{n}\right]^{2}=2 \rho A L \frac{1}{(n \pi)^{2}}[\cos (n \pi)-1]^{2} \quad n=1,2,3, \ldots
\end{aligned}
$$

This theoretical modelling of the beam is the simplest approach to simulate its free transverse vibration and achieve its natural frequencies and mode shapes. However, the beam modelling is not the main subject of this study, and this formulation is only used as a reference for the purpose of comparison with all the results obtained.

\subsection{Experimental modal analysis (EMA) theory}

The first step in the EMA method is the obtaining of Frequency Response Functions (FRF), which can be achieved by applying a specific input and by measuring the output [2]. In practice, a force that is used for exciting the structures must have a sufficient amount of energy in the intended frequency range to stimulate all the vibration modes. One of the most appropriate excitations is random excitation, but in this excitation neither the input signals nor the output signals are periodic; in addition, the measured signals are most often noisy under environmental conditions. Under such conditions, FRFs can be achieved by using the PSD and the Cross Spectral Density (CSD) of the force and the response. By considering the $\mathrm{f}(t)$ as the force signal, the Auto Correlation Function (ACF) of the random force signal can be specified by the following relation:

$$
R_{f f}(\tau)=\lim _{T \rightarrow \infty} \frac{1}{T} \int_{-T / 2}^{T / 2} \mathrm{f}(t) \mathrm{f}(t+\tau) \mathrm{d} t
$$

which can be Fourier transformed as:

$$
S_{f f}(\omega)=\int_{-\infty}^{+\infty} R_{f f}(\tau) e^{-i \omega t} \mathrm{~d} \tau
$$

This is known as Auto Spectral Density (ASD) or Power Spectral Density (PSD), which is also a real and even frequency function. The previous concepts can be developed to simultaneously consider the random force and the random responses. Thus, by considering the $\mathrm{x}(t)$ as the response signal, one can define the Cross Correlation Function (CCF) and the Cross Spectral Density (CSD) function, respectively, as:

$$
\begin{aligned}
& R_{f x}(\tau)=\lim _{T \rightarrow \infty} \frac{1}{T} \int_{-T / 2}^{T / 2} \mathrm{f}(t) \mathrm{x}(t+\tau) \mathrm{d} t \\
& S_{f x}(\omega)=\int_{-\infty}^{+\infty} R_{f x}(\tau) e^{-i \omega t} \mathrm{~d} \tau
\end{aligned}
$$


Using ASD and CSD, FRF can be obtained by the following equation:

$$
H(\omega)=\frac{S_{f x}(\omega)}{S_{f f}(\omega)}
$$

\subsection{Frequency domain decomposition (FDD) theory}

The output-only vibration analysis methods are divided into two parametric and nonparametric groups. The non-parametric methods are used for acquiring the dynamic characteristics of a structure through a series of mathematical operations conducted on the measured data [28]. On the other hand, in the parametric methods, a parametric model is estimated in the time domain, and the dynamic model of the system is extracted directly based on the measured time responses [29]. One of the non-parametric methods is the FDD method presented by Brincker et al. [12]; this method is basically based on the complex mode function extraction and the peak picking method. The procedure is as follows: 1) The matrix of response PSD is calculated. 2) The SVD method is applied. 3) Natural frequencies and mode shapes are obtained.

The principles and governing equations of the FDD method are derived from the relation between the spectra of excitation and the response of a system [30]:

$$
G_{y y}(j \omega)=\bar{H}(j \omega) G_{x x}(j \omega) H^{\top}(j \omega)
$$

in which $G_{x x}$ is the excitation PSD matrix, $G_{y y}$ is the response PSD matrix, $H(j \omega)$ is the FRF matrix, and the overbar and the superscript $\mathrm{T}$ refer to the complex conjugate and transpose matrices, respectively. The FRF matrix can be shown as [2]:

$$
H(j \omega)=\sum_{k=1}^{n} \frac{Q_{k}}{j \omega-\lambda_{k}}+\frac{\bar{Q}_{k}}{j \omega-\bar{\lambda}_{k}}
$$

in which $\lambda_{k}$ is the $k$ th natural frequency, $n$ is the quantity of expected modes, and $Q_{k}$ represents the residual term:

$$
Q_{k}=\phi_{k} \gamma_{k}^{\top}
$$

where $\phi_{k}$ is the $k$ th mode shape vector, and $\gamma_{k}$ is the modal participation factor vector. The combination of Eq. (11) and Eq. (12) results in the following expression [12]:

$$
G_{y y}(j \omega)=\sum_{k=1}^{n}\left[\frac{Q_{k}}{j \omega-\lambda_{k}}+\frac{\bar{Q}_{k}}{j \omega-\bar{\lambda}_{k}}\right] \mathrm{G}_{x x}(j \omega) \sum_{s=1}^{n}\left[\frac{Q_{s}}{j \omega-\lambda_{s}}+\frac{\bar{Q}_{s}}{j \omega-\bar{\lambda}_{s}}\right]^{\mathrm{H}}
$$

where the superscript $\mathrm{H}$ refers to the complex conjugate transpose matrix. Supposing that the excitation has characteristics close to white noise, the PSD matrix will become a diagonal matrix which will be in the form of $G_{x x}(j \omega)=C[I]$. By inserting this relation into Eq. (14) and multiplying the partial fraction factors, together with using the Heaviside partial fraction theorem and simplifying it, one obtains:

$$
G_{y y}(j \omega)=\sum_{k=1}^{n} \frac{A_{k}}{j \omega-\lambda_{k}}+\frac{\bar{A}_{k}}{j \omega-\bar{\lambda}_{k}}+\frac{B_{k}}{-j \omega-\lambda_{k}}+\frac{\bar{B}_{k}}{-j \omega-\bar{\lambda}_{k}}
$$


In this equation, $A_{k}$ is the $k$ th Hermitian residual PSD of response, which is given by:

$$
A_{k}=Q_{k} C\left[\sum_{s=1}^{n} \frac{\bar{Q}_{s}^{\top}}{-\lambda_{k}-\bar{\lambda}_{s}}+\frac{Q_{s}^{\top}}{-\lambda_{k}-\lambda_{s}}\right]
$$

and $B_{k}=A_{k}^{\top}$; Using the orthogonal property of modes, the residual will become $A_{k}=Q_{k} C \bar{Q}_{k}^{\top} / 2 \alpha_{k}$, in which $\alpha_{k}$ is the real part of the $k$ th pole $\lambda_{k}=-\alpha_{k}+j \omega_{k}$. If the damping is low, the residual term is proper for the mode shape and becomes $Q_{k}=\phi_{k} \lambda_{k}$; thus:

$$
A_{k} \propto Q_{k} C \bar{Q}_{k}=\phi_{k} \lambda_{k}^{\top} C \lambda_{k} \phi_{k}^{\top}=d_{k} \phi_{k} \phi_{k}^{\top}
$$

in which $d_{k}$ is a scalar. Finally, the response PSD will be obtained in terms of mode shapes and the system poles as:

$$
G_{y y}(j \omega)=\sum_{k=1}^{n} \frac{d_{k} \phi_{k} \phi_{k}^{\top}}{j \omega-\lambda_{k}}+\frac{\bar{d}_{k} \bar{\phi}_{k} \bar{\phi}_{k}^{\top}}{j \omega-\bar{\lambda}_{k}}
$$

The above relation expresses that a limited number of modes participate in the response at each frequency. In close proximity to natural frequencies, only one mode will play a role in the system response. Thus, the response in this frequency is similar to the mode shape of this frequency. By decomposing the response PSD matrix at each frequency to its singular values and vectors, one can express $G_{y y}$ as:

$$
\left[G_{y y}(\omega)\right]=[U][S][U]^{\mathrm{H}}
$$

in which, $[U]$ is the matrix of singular vectors, and $[S]$ is the matrix of singular values.

Since the singular values are directly related to the factor of modal participation, the number of non-zero singular values represents the number of modes that form the system response at the associated frequency, and the location of the peaks of the first singular value of the PSD matrix corresponds to the natural frequencies of the system; furthermore, the singular vectors, corresponding to the peaks of the first singular values, estimate the mode shape vectors of the system. In the FDD method, the half-power points can be used to acquire the damping ratio, but the degree of accuracy is not acceptable [12].

\section{Laboratory setup and experimental tests}

In order to perform experimental tests, a laboratory beam with a rectangular cross section area is used; properties of the beam are shown in Table 1.

Table 1 Structural material properties.

\begin{tabular}{ccccccc}
\hline $\begin{array}{c}\text { Parts } \\
\text { involved }\end{array}$ & $\begin{array}{c}\text { Material } \\
\text { category }\end{array}$ & $\begin{array}{c}\text { Density } \\
\left(\mathbf{k g} / \mathbf{m}^{\mathbf{3}}\right)\end{array}$ & $\begin{array}{c}\text { Young's } \\
\text { modulus } \\
\mathbf{( G P a )}\end{array}$ & $\begin{array}{c}\text { Poisson's } \\
\text { ratio }\end{array}$ & $\begin{array}{c}\text { Cross } \\
\left.\text { section } \mathbf{( m}^{\mathbf{2}}\right)\end{array}$ & $\begin{array}{c}\text { Length } \\
\mathbf{( m )}\end{array}$ \\
\hline Beam & Aluminium & 2800 & 70 & 0.33 & $0.02 \times 0.01$ & 0.878 \\
\hline
\end{tabular}

The first stage in the modal test is excitation of the structure. Although there are different ways of exciting the structure, two methods, an artificial and a natural (operational) one, are used in this study. A shaker is used to produce artificial excitation, and the force 
generated in this case is white Gaussian noise with a level of $0.7 G V_{R M S}$ in the frequency range from 0 to $2000 \mathrm{~Hz}$. A push-rod (also known as the "stinger" in literature) is used to transmit controlled excitation to the beam, which has high axial stiffness and low bending stiffness. Advantages of this method compared to the method with a rigid connection between the shaker and the system are that any moment of excitation or any rotational loading cannot be imposed on the system. Acoustic waves and natural environmental noise are used for the natural excitation. In the former case, an FM radio frequency signal, which belongs to no special radio channel and which produces white noise is used as excitation, while in the latter case, the noise, deliberately made by people speaking around the setup, is used as excitation. Subsequently, responses of the system are measured. The beam response is measured by seven evenly distributed accelerometers mounted along the beam. Using a data acquisition system including the required hardware and software running on the Windows-based computer, the data are measured for 20 seconds (with a sample rate of $2000 \mathrm{~Hz}$ ) and processed. In Fig. 1, the setup of the real beam used for modal tests is presented. The hardware equipment used in this figure consists of a SINOCERA $8 \mathrm{~N}$ electromagnetic shaker model JZK-2, a B\&K piezoelectric accelerometer $100 \mathrm{mV} / \mathrm{g}$ sensitivity model 4507, a PCB piezoelectric force transducer $2.248 \mathrm{mV} / \mathrm{N}$ sensitivity model 208C03, a National Instrument data acquisition model NI cDAQ-9172, accelerometer modules model NI 9233, a generator module model NI 9269, a B\&K amplifier model 2706, and anti-noise transfer cables.

The force transducer used in the artificial excitation and all seven accelerometers in the setup have small masses, which change only slightly the mass of the base structure. The apparent mass of each transducer seen by the structure in the sensing direction is only about $4 \mathrm{gr}$, which is less than $1 \%$ of the mass of the beam. Therefore, as clarified in section 2 , the reference model is considered to be the mathematical model of a pure beam. The results extracted from the tests are presented and discussed in the following sections of the article.

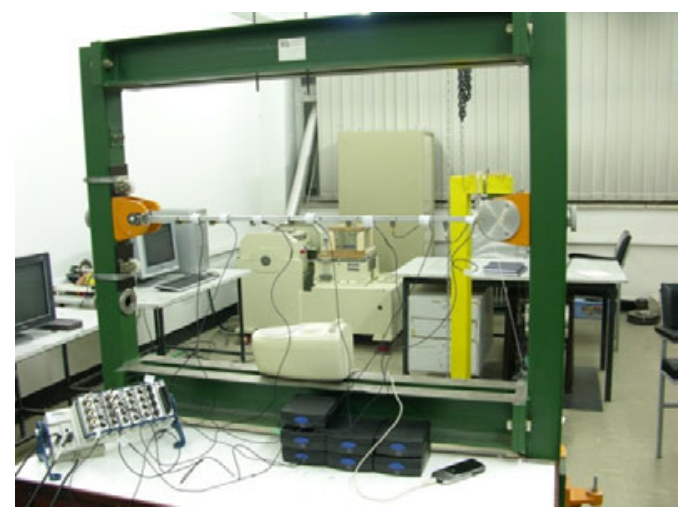

a

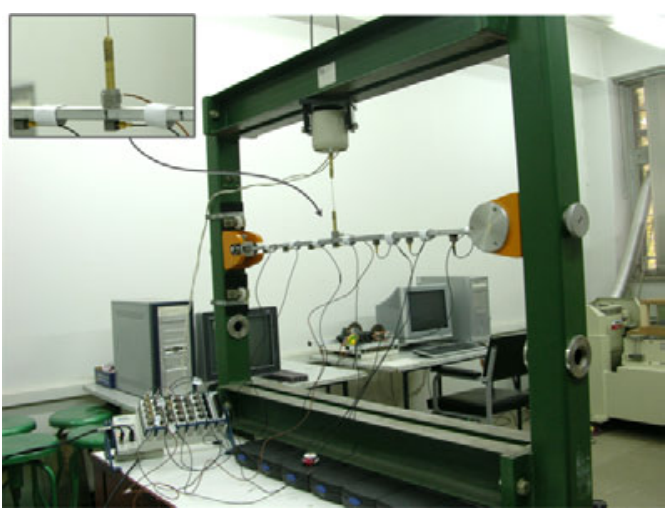

b

Fig. 1 Setup of the beam for modal tests: a) natural excitation, b) artificial excitation.

\section{Results}

\subsection{Natural frequency extraction}

As previously indicated, the FRF values should be acquired to determine the natural frequencies of the beam using the EMA method; for this purpose, Eq. (10) can be employed. Figure 2 shows a diagram of the FRF values obtained from the data recorded by all seven accelerometers. 


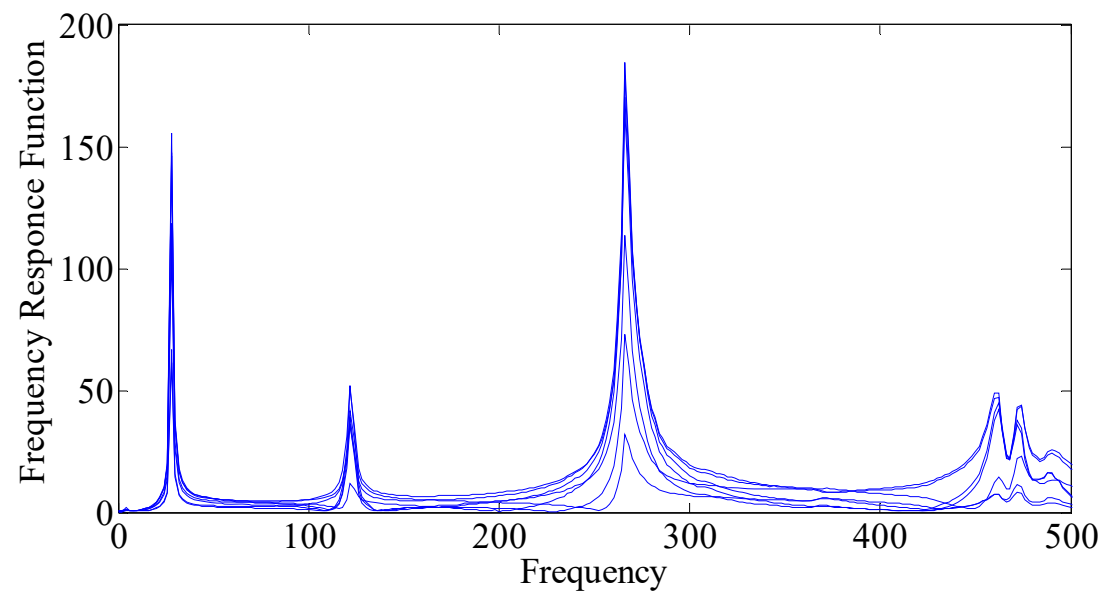

Fig. 2 Diagram of the FRF values of measured signals.

In the next step, the modal parameters can be extracted using the EMA method. In the analysis, the Rational Fraction Polynomial (RFP) method [7] is used to obtain the results which are presented in the following sections of the article.

In the case of the OMA method, the FDD method is applied in order to determine the natural frequencies. Firstly, diagrams of singular values of response PSD are drawn for all frequencies. As stated above, peaks of these diagrams correspond to the natural frequencies of the system. The diagrams of normalized singular values for different types of excitation sources (i.e. shaker, acoustic waves, and environmental noises) are shown in Fig. 3, 4, and 5.

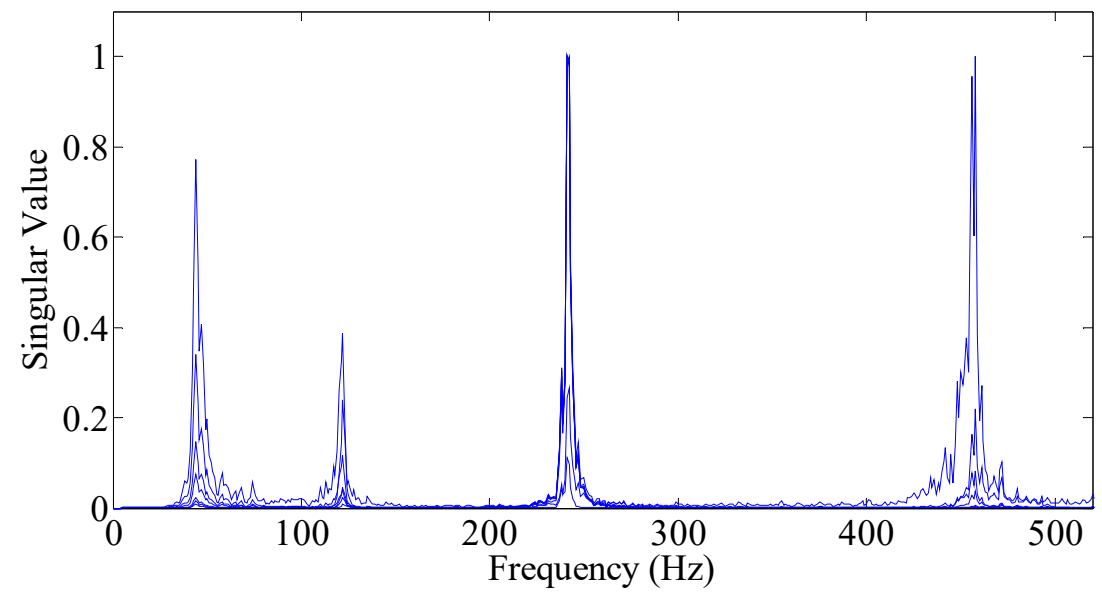

Fig. 3 Singular value diagram of the PSD matrix for the system excited by a shaker.

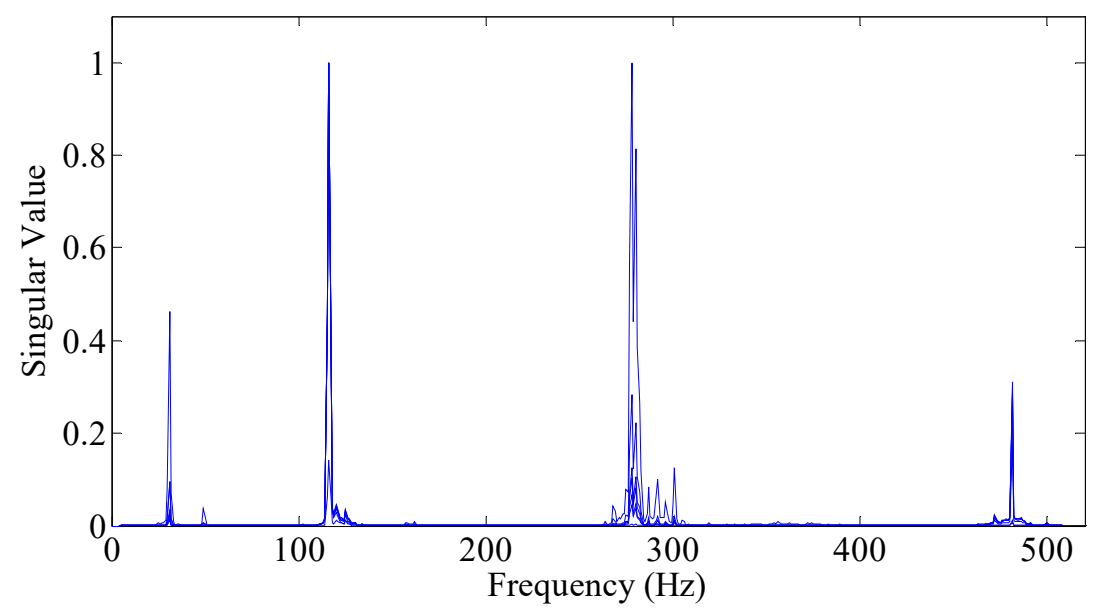

Fig. 4 Singular value diagram of the PSD matrix for the system excited by acoustic waves. 


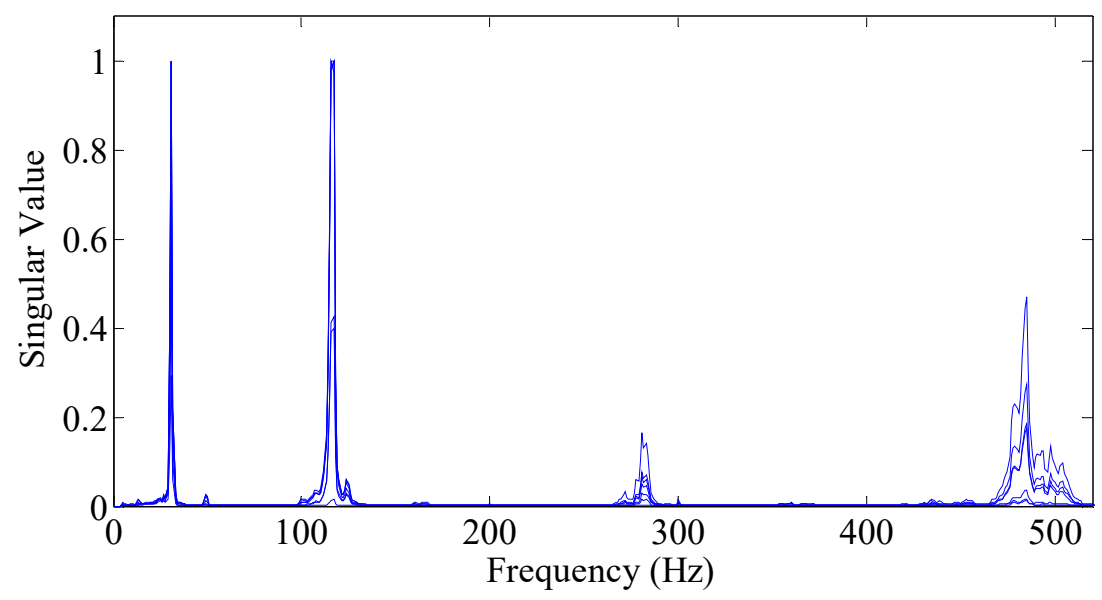

Fig. 5 Singular value diagram of the PSD matrix for the system excited by environmental noise.

In Table 2, average values of determined natural frequencies (i.e. the average of the frequencies obtained from the data recorded by all seven accelerometers) are shown for all proposed methods. The analytical values of natural frequencies are also reported as reference values for the purpose of comparison. In addition, the effective modal mass values obtained through Eq. (5) are presented as percentages. It should be noted that the effective modal mass values (which are directly related to the modal participation factor) would provide a good insight into the calculation and averaging of errors of different results compared with analytical reference values.

Table 2 Obtained natural frequencies in comparison with analytical reference values $(\mathrm{Hz})$.

\begin{tabular}{ccccccc}
\hline Mode shape & $\begin{array}{c}\text { Analytical } \\
\text { reference } \\
\text { value }\end{array}$ & EMA & $\begin{array}{c}\text { OMA by } \\
\text { shaker }\end{array}$ & $\begin{array}{c}\text { OMA by } \\
\text { acoustic } \\
\text { waves }\end{array}$ & $\begin{array}{c}\text { OMA by } \\
\text { environmental } \\
\text { noise }\end{array}$ & $\begin{array}{c}\text { Effective } \\
\text { modal } \\
\text { mass (\%) }\end{array}$ \\
\hline First mode & 29.38 & 28.00 & 44.00 & 30.00 & 30.00 & 81.06 \\
Second mode & 117.51 & 122.00 & 122.00 & 116.00 & 116.00 & 0 \\
Third mode & 264.40 & 266.00 & 242.00 & 278.00 & 282.00 & 9.01 \\
Fourth mode & 470.04 & 462.00 & 456.00 & 482.00 & 484.00 & 0 \\
\hline
\end{tabular}

As mentioned previously in section 3, a shaker was used to artificially excite the system by ideal white Gaussian noise, and a push-rod was used to transmit the generated force without any other external effects. Contrary to expectations, significant errors are observed in the results of OMA by shaker compared to the analytical reference values (see Table 2). On the other hand, the results presented in Table 2 show that the effective modal mass of even modes is zero while it is $81.06 \%$ for the first mode, which means that the first mode is critical for the simply supported beam and can be readily excited by base excitation. The results are compared and discussed in the following sections.

\subsection{Mode shape extraction}

Around the natural frequencies of the system, the corresponding mode becomes dominant in the response of the system and makes a great contribution to it. Thus, the response in this frequency is similar to the mode shape of the associated frequency; furthermore, the singular vectors corresponding to the peaks of the first singular values estimate the mode shape vectors. In this regard, to check the precision and accuracy of the methods used to estimate the mode shapes of the beam, the results extracted for different states are depicted in Fig. 6 to 9. 


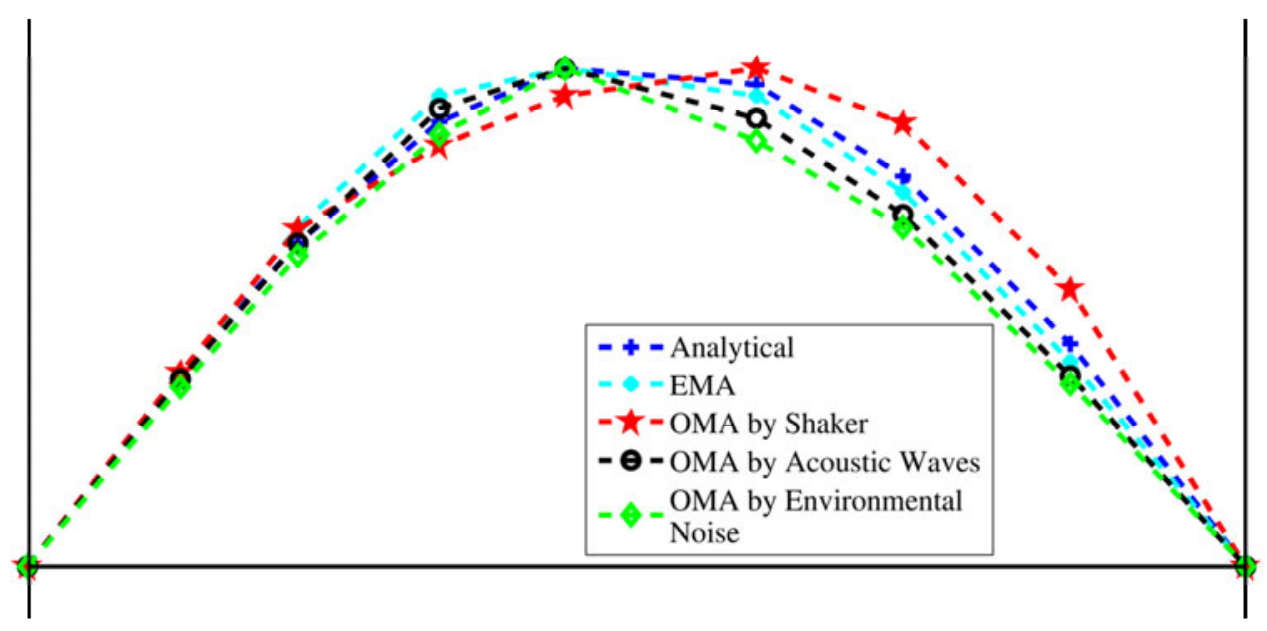

Fig. 6 Estimated mode shapes of the beam in the first vibration mode.

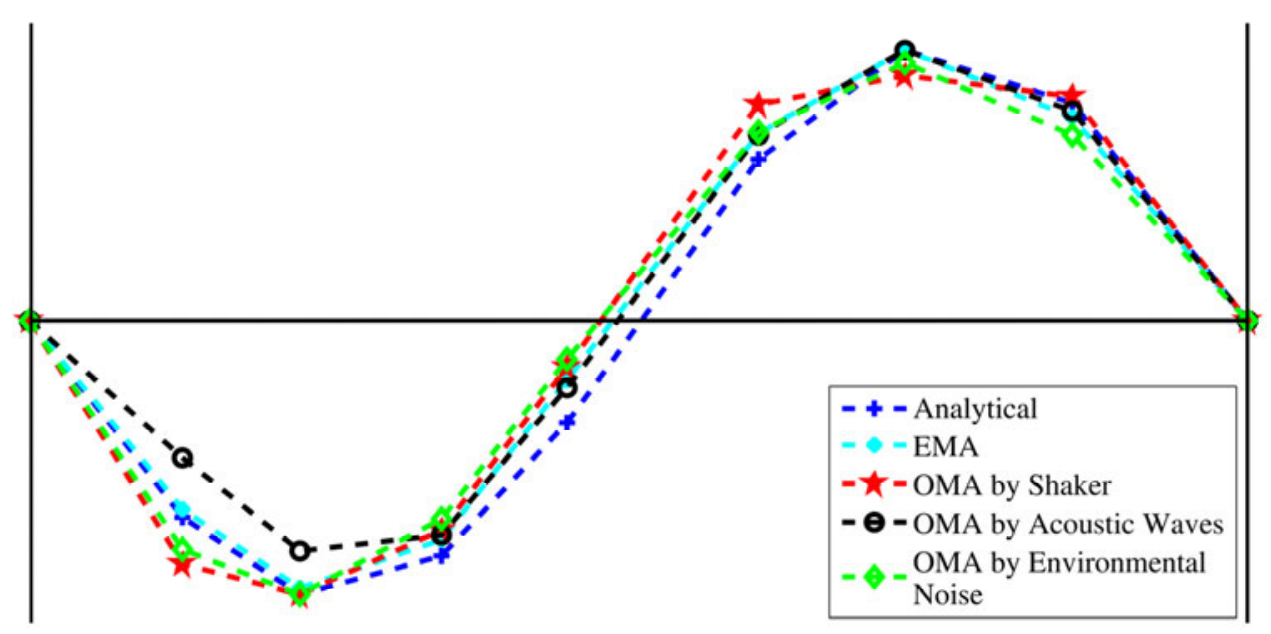

Fig. 7 Estimated mode shapes of the beam in the second vibration mode.

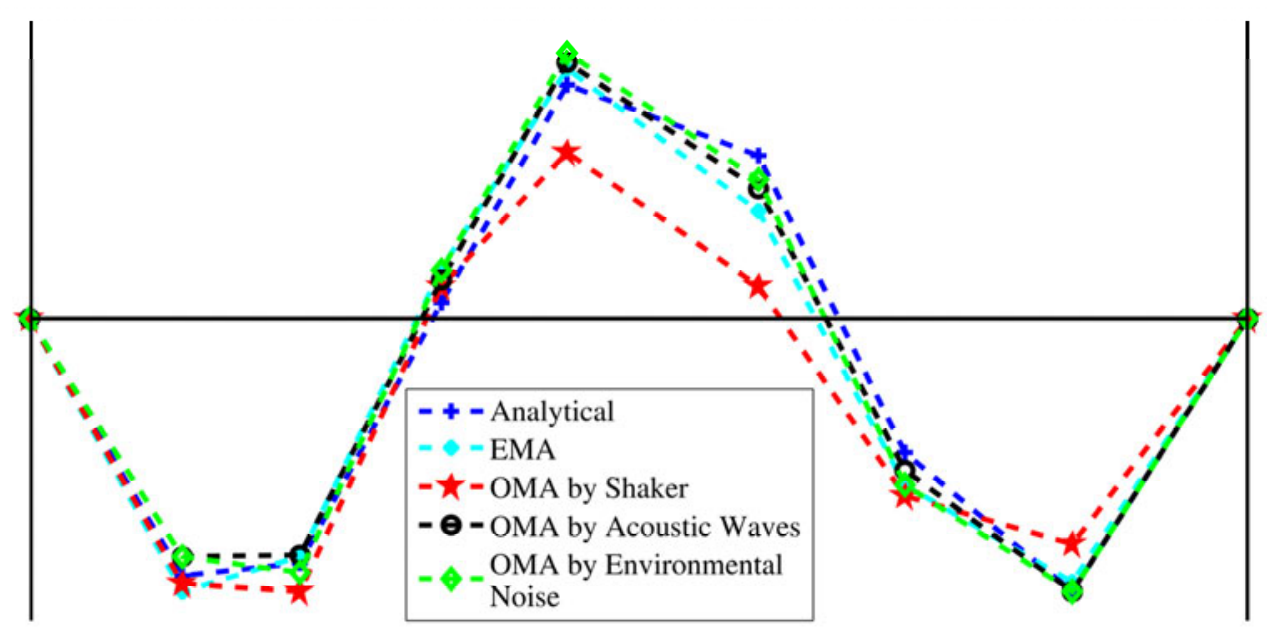

Fig. 8 Estimated mode shapes of the beam in the third vibration mode. 


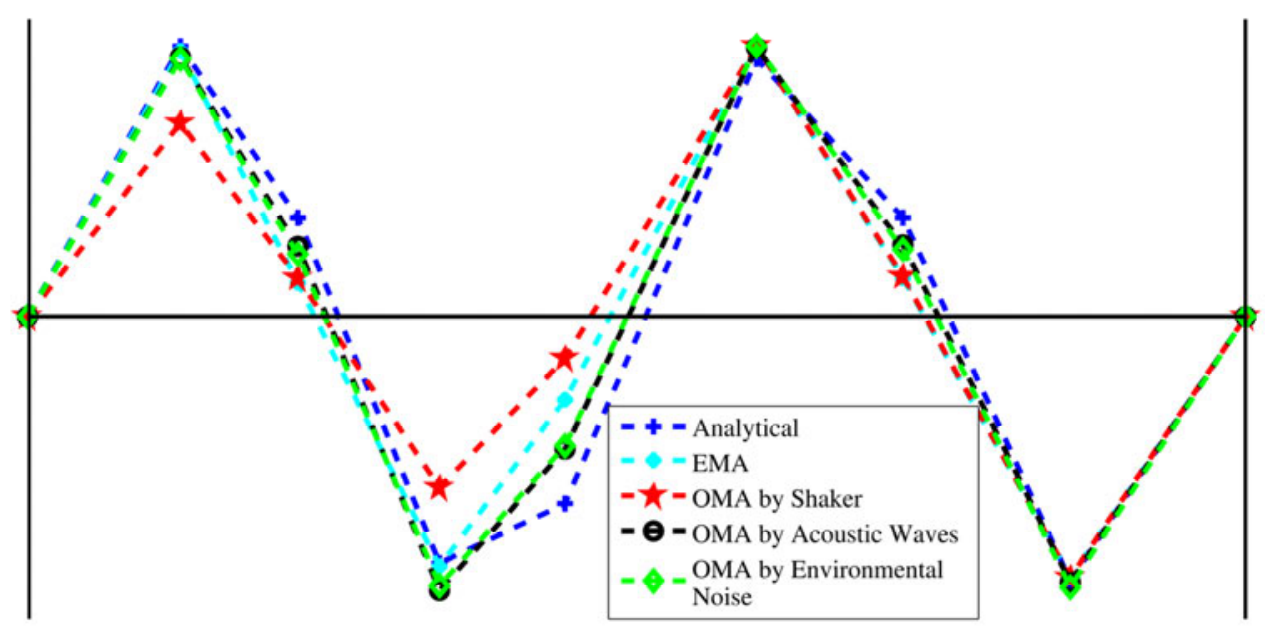

Fig. 9 Estimated mode shapes of the beam in the fourth vibration mode.

In Fig. 6 to 9, one can immediately note a great error in the results of OMA by shaker compared to the analytical reference mode shapes and other results, such that an obvious asymmetry can be seen in odd mode shapes. Since the excitation values are not included in the OMA calculations, this consequence may originate from the connection between the shaker in the middle of the beam and the beam. Clearly, the aforementioned connection is a nodal point in the even modes and an anti-nodal point in the odd modes; therefore, odd modes are more affected than even modes, and the above-mentioned error is greater in odd modes.

\subsection{Comparison of results}

In this section, the estimated natural frequencies and extracted mode shapes reported in the previous sections are compared to each other. Table 3 shows the errors in the values of natural frequencies (experimentally estimated) with respect to the analytical reference values, taking into account the effective modal mass values as weighting factors (see Table 2).

Table 3 Relative errors in the values of natural frequencies with respect to effective modal mass values.

\begin{tabular}{ccccc}
\hline & \multicolumn{4}{c}{ Relative errors (\%) } \\
\cline { 2 - 5 } Different Cases & EMA & $\begin{array}{c}\text { OMA by } \\
\text { shaker }\end{array}$ & $\begin{array}{c}\text { OMA by } \\
\text { acoustic waves }\end{array}$ & $\begin{array}{c}\text { OMA by } \\
\text { environmental } \\
\text { noise }\end{array}$ \\
\hline First mode & 3.8 & 40.35 & 1.72 & 1.72 \\
Second mode & 0 & 0 & 0 & 0 \\
Third mode & 0.05 & 0.76 & 0.46 & 0.6 \\
Fourth mode & 0 & 0 & 0 & 0 \\
Average error & 0.96 & 10.28 & 0.55 & 0.58 \\
\hline
\end{tabular}

As can be seen from the results presented in Table 3, the average error of all experimental approaches is less than $1 \%$ except for OMA by shaker, which is $10.28 \%$. In order to figure out the cause of this unexpected big error, spectral characteristics of the input artificial excitation are investigated. The PSD values of the measured input excitation are obtained for this purpose and shown in the diagram in Fig. 10. It is obvious that the system is excited in all desired frequency ranges, but it is immediately noticeable that the amplitude of the PSD values of the input excitation is considerably increased around certain frequencies, 
i.e. the natural frequencies of odd modes. As it has been mentioned previously, the connection between the shaker and the beam is the anti-nodal point of the odd vibrational modes of the beam; therefore, the excitation signal is affected when the beam is excited around the associated natural frequencies. This problem is not observed at other frequencies, including the natural frequencies of even modes (with respect to the connection between the shaker and the beam at the nodal point of the associated modes), which is consistent with previous observations. Consequently, since the input excitation is not involved in the OMA calculations, considerable errors are observed in the estimated odd natural frequencies of the beam. Therefore, it seems that other factors, such as the working frequency range, the modal participation of the system in operation conditions, and the connection between the shaker and the system, should be taken into account in the analysis of this approach.

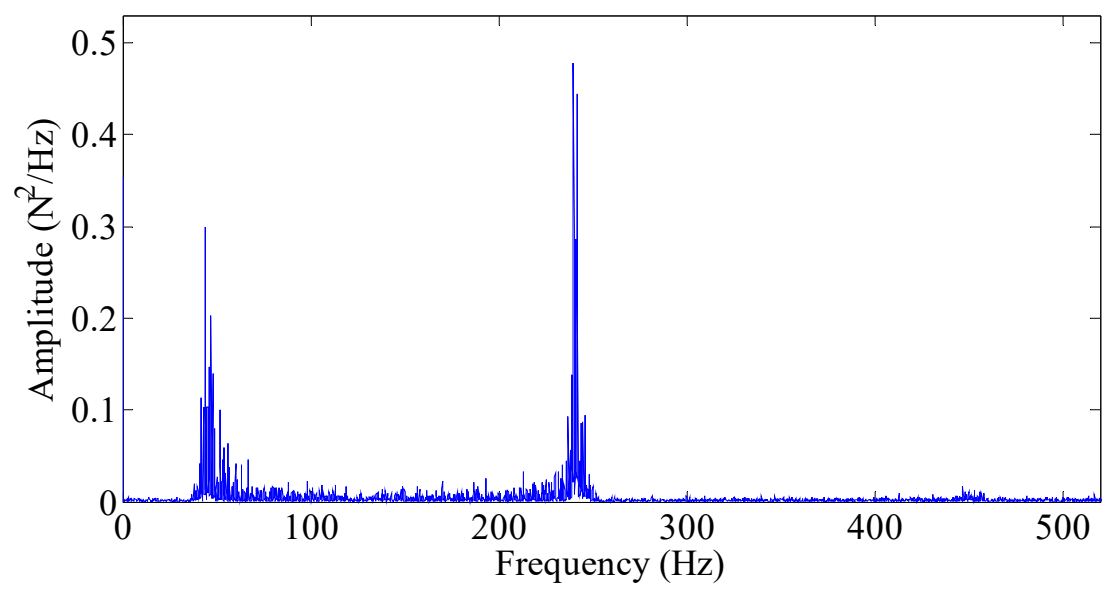

Fig. 10 Diagram of the PSD values of input artificial excitation.

To compare the mode shapes extracted by the analytical and experimental methods, and to evaluate the similarity between the two groups of mode shapes, the Modal Assurance Criterion (MAC) can be employed [31]. It provides the proximity between the two groups of mode shapes, $\{\phi\}$ and $\{\psi\}$ :

$$
\operatorname{MAC}\left(\{\phi\}_{i},\{\psi\}_{j}\right)=\frac{\left|\varphi_{i}^{\top} \psi_{j}\right|^{2}}{\left|\varphi_{i}^{\top} \varphi_{i}\right| \times\left|\psi_{j}^{\top} \psi_{j}\right|}
$$

The MAC values which are generated by this equation fluctuate between 0 and 1 ; the unitary value indicates a perfect correlation between the two mode shapes, but zero means that there is no resemblance between them. In practice, values greater than 0.9 indicate acceptable correspondence between the two mode shapes. It should be noted that this criterion evaluates the similarity between the mode shapes, but it does not investigate the orthogonality between them. Comparisons of the mode shapes which have already been extracted from different methods are shown in Fig. 11 to 14. 

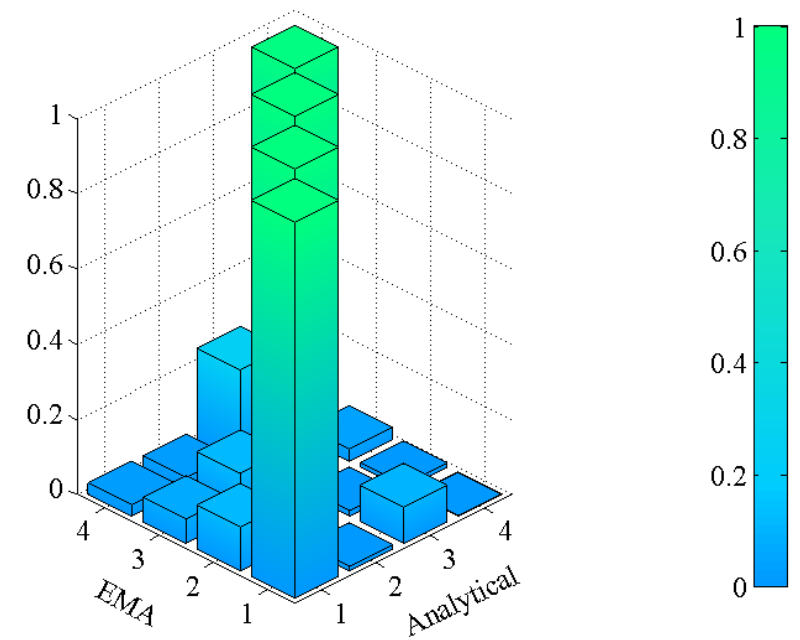

Fig. 11 Comparison between the two groups of estimated mode shapes (Analytical method and EMA) by employing MAC.
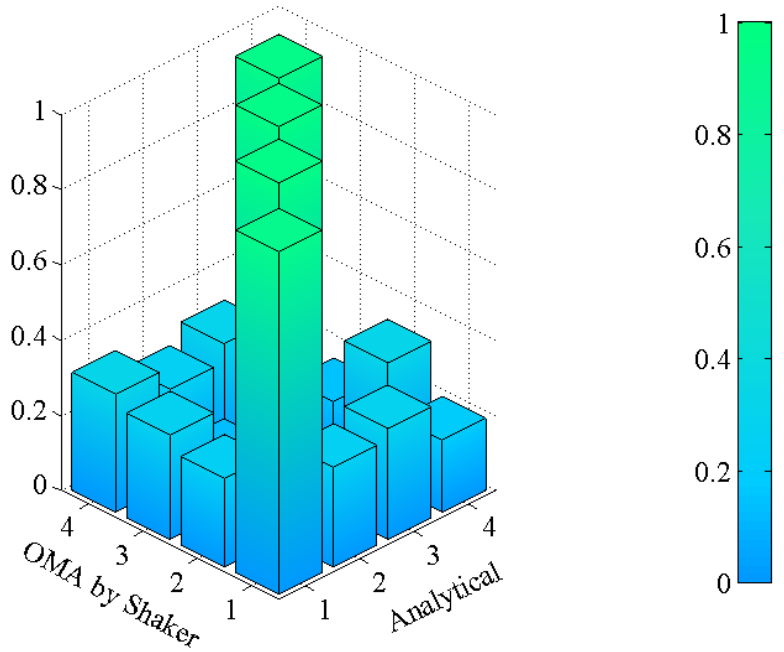

Fig. 12 Comparison between the two groups of estimated mode shapes (Analytical method and OMA by shaker) by employing MAC.
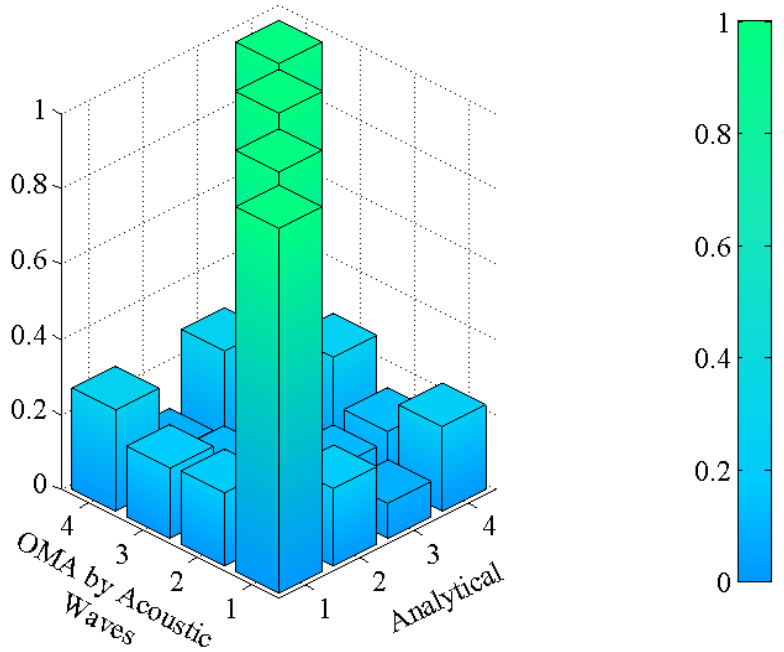

Fig. 13 Comparison between the two groups of estimated mode shapes (Analytical method and OMA by acoustic waves) by employing MAC. 

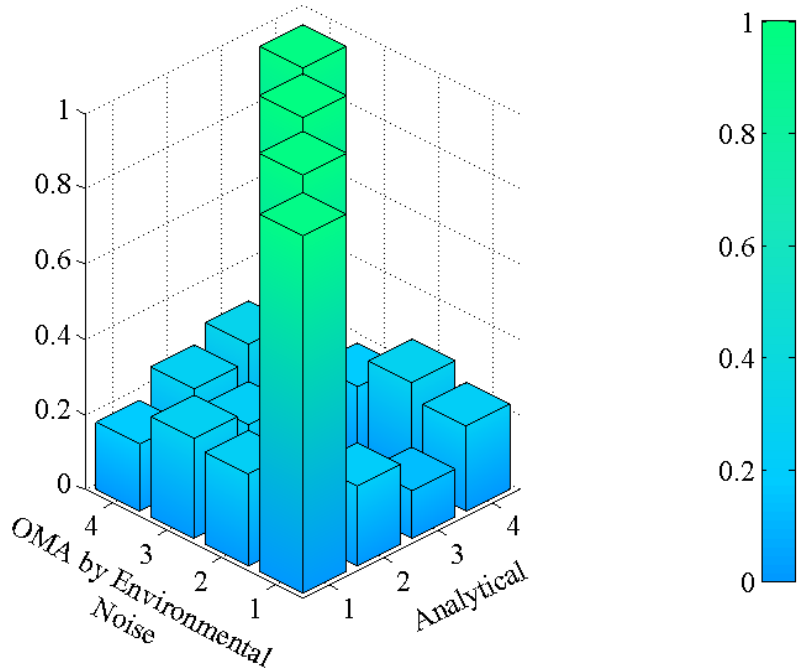

Fig. 14 Comparison between the two groups of estimated mode shapes (Analytical method and OMA by environmental noise) by employing MAC.

As can be clearly seen in Fig. 11 to 14 , the error in the results of the EMA method compared to the results of the analytical method is less than in the OMA method under different conditions; the average value of the diagonal elements of the obtained MAC numbers is 0.99 , and the average value of the off-diagonal elements is 0.06 .

Comparing the results of the OMA method under different conditions indicates that the acoustic excitation achieves more accurate mode shapes than the other two excitations; the average value of the diagonal elements and the off-diagonal elements are 0.98 and 0.18 , respectively; however, these values based on the environmental noise excitation are 0.96 and 0.21 , and those based on the shaker excitation are 0.93 and 0.23 , respectively.

\section{Conclusion}

An experimental study of the effects of excitation types on the performance of the OMA method in predicting the modal characteristics of structures was carried out in our research. For that purpose, a laboratory scale beam was mathematically modelled based on the EulerBernoulli theory, and the EMA method was adopted. The results of the EMA method were in good agreement with the analytical reference values. That observation showed that the experimental setup was properly constructed, while the instruments attached to the beam, especially the probe, i.e. a flexible push-rod, connecting the shaker to the base structure, have a minimal effect on the mass and the flexural rigidity of the beam.

Once the EMA and analytical results were obtained, the OMA experiments were carried out to study the effect of excitation types on the results. The OMA analysis was based on the FDD and SVD approaches. In the FDD method, the quality of the singular value diagrams of PSD matrices, which were obtained for different types of excitation, is important; therefore, the results depend on these diagrams. After reviewing and investigating the obtained results, the following was observed:

- Excitation by acoustic waves for detecting the natural frequencies in the OMA method had desired results, with the average error of approximately $0.55 \%$. Surprisingly, the environmental noise excitation also had acceptable results, with the average error of approximately $0.58 \%$. However, the average error of the shaker excitation was about $10.28 \%$ compared to the analytical method.

- Although the results based on EMA demonstrated that the experimental setup was properly constructed and although it was expected that the application of the ideal white 
Gaussian noise via a shaker would lead to acceptable results with small errors, the results of repeated experiments were unexpected. Considering that the excitation was directly applied to the beam with a probe and that this excitation force was not taken into account in vibration analyses, there was inaccuracy at certain natural frequencies obtained from the OMA method with respect to the connection between the shaker and the beam, and some precautions should be taken when the OMA method is used in the experiments with artificial excitation by a shaker.

- Acceptable concordance was obtained between the mode shapes estimated by the analytical and experimental methods. For a more detailed comparison of the results, the MAC numbers were extracted from the test and the analytical method results. The best result was obtained based on the EMA method. By comparing the extracted MAC numbers for the OMA method, the mode shapes extracted by acoustic excitation were closer to the analytical mode shapes.

- Finally, it was found that the acoustic excitation achieves better results with smaller errors compared to the other conditions in the OMA method using the FDD and SVD techniques; furthermore, the environmental noise excitation achieves acceptable results with a slightly greater error.

\section{REFERENCES}

[1] Ljung L. System Identification: Theory for the user. Prentice-hall; 1987

[2] Ewins DJ. Modal Testing: Theory, Practice and Application. Research Studies Press, 2nd edition; 2000.

[3] Peeters B, De Roeck G. Stochastic system identification for operational modal analysis: a review. Journal of Dynamic Systems, Measurement, and Control. 2001 Dec 1; 123(4):659-667, https://doi.org/10.1115/1.1410370

[4] Brown DL, Allemang RJ, Zimmerman R, Mergeay M. Parameter estimation techniques for modal analysis. SAE Technical paper; 1979 Feb 1, https://doi.org/10.4271/790221

[5] Ibrahim SR, Mikulcik, EC. A time domain modal vibration test technique. The Shock and Vibration Bulletin. 1973; 43(4): 21-37, https://doi.org/10.1177/058310247400600615

[6] Ibrahim SR, Mikulcik EC. The experimental determination of vibration parameters from time responses. The Shock and Vibration Bulletin. 1976 Aug; 46(5):187-196.

[7] Richardson MH, Formenti DL. Parameter estimation from frequency response measurements using rational fraction polynomials. InProceedings of the 1st international modal analysis conference $1982 \mathrm{Nov}$ 8 (Vol. 1, pp. 167-186). Union College Schenectady, NY.

[8] James GH, Carne TG, Lauffer JP. The natural excitation technique for modal parameter extraction from operating structures. The International Journal of Analytical and Experimental Modal Analysis. 1995; 10(4):260-277.

[9] Caicedo JM. Practical guidelines for the natural excitation technique (NexT) and the eigensystem realization algorithm (ERA) for modal identification using ambient vibration. Experimental Techniques. 2011 Jul 1; 35(4):52-58, https://doi.org/10.1111/j.1747-1567.2010.00643.x

[10] Peeters B, De Roeck G. Reference-based stochastic subspace identification for output-only modal analysis. Mechanical systems and signal processing. 1999 Nov 30; 13(6):855-878, https://doi.org/10.1006/mssp.1999.1249

[11] Brincker R, Zhang L, Andersen P. Modal identification from ambient responses using frequency domain decomposition. InProceeding the 18th International Modal Analysis Conference (IMAC), San Antonio, Texas 2000 Feb.

[12] Brincker R, Zhang L, Andersen P. Modal identification of output-only systems using frequency domain decomposition. Smart materials and structures. 2001 Jun; 10(3):441-445, https://doi.org/10.1088/0964$1726 / 10 / 3 / 303$

[13] Bendat JS, Piersol AG. Random data: analysis and measurement procedures. John Wiley \& Sons; 2011 Sep 20.

[14] Bendat JS, Piersol AG. Engineering applications of correlation and spectral analysis. New York, WileyInterscience, 1980. 
[15] Ventura CE, Horyna T. Structural assessment by modal analysis in Western Canada. In Proceedings-Spie the international society for optical engineering 1997 (pp. 101-105). Spie international society for optical.

[16] Brincker R, Ventura C, Andersen P. Damping estimation by frequency domain decomposition. In 19th International Modal Analysis Conference 2001 Feb 5 (pp. 698-703).

[17] Brincker R, Kirkegaard PH. Special issue on operational modal analysis. Mechanical systems and signal

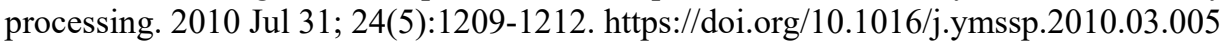

[18] Zhang L, Wang T, Tamura Y. A frequency-spatial domain decomposition (FSDD) method for operational modal analysis. Mechanical systems and signal processing. 2010 Jul 31; 24(5):1227-1239, https://doi.org/10.1016/j.ymssp.2009.10.024

[19] Magalhães F, Cunha Á, Caetano E, Brincker R. Damping estimation using free decays and ambient vibration tests. Mechanical Systems and Signal Processing. 2010 Jul 31; 24(5):1274-1290, https://doi.org/10.1016/j.ymssp.2009.02.011

[20] Yan WJ, Ren WX. Operational modal parameter identification from power spectrum density transmissibility. Computer-Aided Civil and Infrastructure Engineering. 2012 Mar 1; 27(3):202-217, https://doi.org/10.1111/j.1467-8667.2011.00735.x

[21] Araújo IG, Laier JE. Operational modal analysis using SVD of power spectral density transmissibility matrices. Mechanical Systems and Signal Processing. 2014 May 3; 46(1):129-145, https://doi.org/10.1016/j.ymssp.2014.01.001

[22] Yan WJ, Ren WX. An Enhanced Power Spectral Density Transmissibility (EPSDT) approach for operational modal analysis: Theoretical and experimental investigation. Engineering Structures. 2015 Nov 1; 102:108-119, https://doi.org/10.1016/j.engstruct.2015.08.009

[23] Kudu FN, Uçak Ş, Osmancikli G, Türker T, Bayraktar A. Estimation of damping ratios of steel structures by Operational Modal Analysis method. Journal of Constructional Steel Research. 2015 Sep 30; 112:6168, https://doi.org/10.1016/j.jcsr.2015.04.019

[24] Wang T, Celik O, Catbas FN, Zhang LM. A frequency and spatial domain decomposition method for operational strain modal analysis and its application. Engineering Structures. 2016 May 1; 114:104-112, https://doi.org/10.1016/j.engstruct.2016.02.011

[25] Malekjafarian A, OBrien EJ. Identification of bridge mode shapes using short time frequency domain decomposition of the responses measured in a passing vehicle. Engineering Structures. 2014 Dec 15; 81:386-397, https://doi.org/10.1016/j.engstruct.2014.10.007

[26] Rao SS. Vibration of continuous systems. John Wiley \& Sons; 2007 Feb 9.

[27] Genta G. Vibration of structures and machines: practical aspects. Springer Science \& Business Media; 2012 Dec 6.

[28] Wenzel H, Pichler D. Ambient vibration monitoring. John Wiley \& Sons; 2005 Dec 13.

[29] Zhang L, Brincker R. An overview of operational modal analysis: major development and issues. In 1st international operational modal analysis conference 2005 Apr 26 (pp. 179-190). https://doi.org/10.1002/9781118535141

[30] Brandt A. Noise and vibration analysis: signal analysis and experimental procedures. John Wiley \& Sons; 2011 Mar 29.

[31] Allemang RJ, Brown DL. A correlation coefficient for modal vector analysis. In Proceedings of the 1st international modal analysis conference 1982 Nov 8 (Vol. 1, pp. 110-116). SEM, Orlando.

$\begin{array}{ll}\text { Submitted: } & 17.02 .2018 \\ \text { Accepted: } & \quad 06.5 .2019\end{array}$
Sina Varahram

E-mail: s.varahram90@ms.tabrizu.ac.ir

Peyman Jalali

Morteza H. Sadeghi

Saeed Lotfan

Vibration and Modal Analysis Research

Lab, Faculty of Mechanical Engineering,

University of Tabriz, Tabriz, Iran 